\title{
Introduction to the Minitrack on Smart (City) and Data Streaming Application Development: Challenges and Experiences
}

\author{
Sandra Gesing \\ University of Illinois \\ Discovery Partners Institute \\ sgesing@uillinois.edu
}

\author{
Peter Salhofer \\ FH Joanneum \\ peter.salhofer@fh-joanneum.at \\ Charlie Catlett \\ University of Illinois Discovery \\ Partners Institute \\ ccatlett@illinois.edu
}

\author{
Cezary Mazurek \\ Poznan Supercomputing and \\ Networking Center \\ mazurek@psnc.pl
}

\begin{abstract}
Building Smart (City) Applications and data streaming have been research domains for several years now. COVID-19 has accelerated digitalization for smart cities in topics such as remote monitoring of infrastructure. While many people started to work from home and many moved more to rural areas during the pandemic, we are convinced that urbanization will return with the need of planning for smart transportation and support of a lifestyle that focuses on community and sustainability. This minitrack features the concepts and ideas of Smart Applications and data streaming applications, their implementations, especially from a software engineering point of view. Submissions to this minitrack include presentations of architectures, frameworks, platforms and infrastructures as well as success stories of implementations.
\end{abstract}

\section{Introduction}

Software challenges for smart applications are broad, not only regarding the breadth of the community, but also to the breadth of topics. A specific focus of this minitrack is on how to deal with the details of scenarios like handling huge data sets, high usage loads, complex event processing on real-time data streams, sharing data between applications, security, etc. Submissions about disruptive technologies are welcome, which influence Smart (City) Applications development, e.g., 5G networks, Spatial Computing, Blockchain, Conversational Computing and Autonomous Vehicles.

Following topics are in the scope of the minitrack:
- System Architectures for Smart Application and Industry 4.0

- Best practices and Key Success Factors in Smart Application Development

- Platforms for Smart Applications

- Smart Applications for Smarter Government

- Data-hubs and their roles in Smart Application Developments

- Easy-to-use end-to-end solutions for Smart Applications

- Web services for Smart Applications

- Real-time data analytics and machine learning in practice

- Successful Smart Application Project Management

- Infrastructures for Smart Applications

- Securing Smart Applications and Sensor Networks

- Promoting Smart Applications

- Digital Twin technology for Smart Cities.

\section{Accepted Papers}

We received five submissions with a broad range of excellent topics such as parking vacancy detection to fusion of social media and sensor data for analyzing COVID-19 restrictions to pillars of digital transformation of smart cities. We accepted the following three papers out of the five.

"Deep Learning Based Parking Vacancy Detection for Smart Cities" tackles the parking shortage that occurs in large cities. Drivers cruising in search of a parking space directly translate into frustration, traffic congestion, and excessive carbon emission. The authors 
present a deep learning-based parking space notification system to inform drivers of new parking availabilities and re-occupancy of the freed spaces. The PSN system uses a unique adaptation of the IoU concept to track the changes of vehicle positions in a video stream from a CCTV camera installed at a private parking lot and publicly available YouTube videos. The PSN system successfully captured all new parking vacancies arising from leaving vehicles with no false positive detections. Prompt notification messages were sent to users via cloud messaging services.

I. Gudmundsson and G. Falco tackle the challenge of monitoring smart grid infrastructure. Their use case takes place in harsh operating conditions in extreme, remote environments in the highlands of Iceland. Current methods for monitoring such critical infrastructure includes manual inspection, static video analysis (where connectivity is available) and unmanned aerial vehicle (UAV) inspection. UAVs offer certain inspection efficiencies; however, challenges persist given the time and UAV operator skill required. Collaborating with Landsnet, the Icelandic smart grid operator, they apply convolutional neural networks for image processing to detect smart grid transmission infrastructure and modify the resulting computer vision (CV) model to function on the edge of a UAV. Their real-time $\mathrm{CV}$ model delivers decision insight on the UAV edge and enables autonomous flight path planning for use in smart grid inspection. The approach is transferable to other smart city applications that could benefit from edge-based monitoring and inspection.
"Technology pillars for digital transformation of cities based on open software architecture for end2end data streaming" analyzes the pillars of urban digital infrastructure. The authors describe the need for a general-purpose infrastructure in the city that improves the quality of life with respect to various types of socioeconomic activity of residents. The digital aspect of fostering this kind of activity is linked to a very dynamic and ubiquitous element of technology, and the quality and stability of solutions based on advanced technology is a key parameter of the quality of life and development that a city should provide to its residents. This pace of technology development means that the environment and cities are subject to a process of continuous digital transformation. The authors give three examples of platforms designed for City of Poznan and conclude with their findings on fostering community and sustainability.

\section{Conclusion}

The papers present the breadth of applications in smart technologies and show also the shift to focusing more on sustainable and community-oriented solutions. We are looking forward to lively discussions at HICSS55 with the authors and audience. We plan to continue this minitrack in the future and invite authors on topics around software and success stories for smart applications and data streaming. 